videotaped and analysed with a validated observation system allowing quantification of the intensity of physical contacts (PC). Five levels of intensity were coded. Level 1 represents the lowest intensity, and level 5 the highest. Different types of PC such as slashing and hooking were also observed. Multivariate Poisson's regression analyses were performed to compare games between the two cohorts. The results provided a body contact incidence rate per team game. Rates were adjusted for the game period, rink zone, and score difference.

Results A total of 5,610 incidences of body contact with the trunk and 3,429 other types of body contact were observed. Very light intensity (Level 1) trunk contact was more frequent in Québec City (adjusted incidence RR [ARR]: 1.71; 95\% CI: 1.282.29). Hooking (ARR: 2.18; 95\% CI: 1.42-3.32) and slashing (ARR: 3.35 ; 95\% CI: 1.31-8.58) were more frequent in Calgary. Body contacts were made more often by defensive players (ARR: 1.28; 95\% CI: 1.03-1.58) and on puck carriers (ARR: 1.47; 95\% CI: 1.02-2.12) in Calgary.

Conclusions Results suggest that players' behaviours differ between players in leagues in which $\mathrm{BC}$ was permitted at age 11 compared to leagues in which $\mathrm{BC}$ was delayed until age 13 .

\section{THE INCIDENCE OF STRESS FRACTURES IN AMERICAN COLLEGIATE ATHLETES}

${ }^{1}$ Katherine Rizzone, ${ }^{2}$ Zachary Kerr, ${ }^{3}$ Kathryn Ackerman, ${ }^{4}$ Karen Roos, ${ }^{5}$ Aristarque Djoko, ${ }^{6}$ Thomas Dompier. 'University of Rochester Medical Centre; ${ }^{2}$ NCAA Injury Surveillance Program Datalys Centrefor Sports Injury Research; ${ }^{3}$ Boston's Children Hospital, Harvard Medical School; ${ }^{4}$ University of North Carolina at Chapel Hill; ${ }^{5}$ Datalys Centrefor Sports Injury Research; ${ }^{6}$ University of South Carolina

\subsection{6/injuryprev-2016-042156.747}

Background Stress fractures are common sports-related injuries; female and endurance athletes are known to be at a higher risk than the general athlete population. There are thousands of collegiate athletes in the United States competing in high-impact sports, but no large study has been conducted to determine the incidence of stress fractures in this population. Our objective was to calculate the incidence of stress fractures in National Collegiate Athletic Association athletes and investigate epidemiological trends in specific subgroups of athletes.

Methods Data were analysed from the NCAA Injury Surveillance Program from 2009-2010 to 2014-2015 academic years.

Results A total of 747 stress fractures resulting in time loss were reported during 18,054,757 athletic exposures (AE) for an overall rate of $0.53 / 10,000 \mathrm{AE}$ (95\% CI: $0.50,0.57)$. The rate of fracture was higher overall among female athletes $(\mathrm{RR}=2.06 ; 95 \%$ CI: 1.71, 2.47). Female cross country runners had higher fracture rates than males runners $(\mathrm{RR}=1.77 ; 95 \% \mathrm{CI}: 1.05,2.98)$, but this was also noted in female versus male soccer $(\mathrm{RR}=1.69$; $95 \% \mathrm{CI}: 1.09,2.63)$ and basketball $(\mathrm{RR}=1.69 ; 95 \% \mathrm{CI}: 1.26$, 2.28) players. The pre-season rate was larger than the regular-/ post-season rate $(\mathrm{RR}=3.27 ; 95 \% \mathrm{CI}: 2.83,3.78)$. The most common stress fracture locations were the metatarsals $(38.8 \%$, $\mathrm{N}=290)$, tibia $(20.7 \%, \mathrm{~N}=155)$, and the lower back/lumbar spine/pelvis $(13.4 \%, \mathrm{~N}=100)$ and $22.5 \%(\mathrm{~N}=165)$ of stress fractures were recurrent.

Conclusion Females had higher fracture rates of stress fractures than males; but future work needs to focus on improved screening tools for female athletes for all sports as our results found a gender disparity in additional sports from the well-known crosscountry athletes. A fifth of fractures are recurrent, potentially highlighting the need to reassess post-injury return to play policies.

\section{\begin{tabular}{l|l}
748 & IMPLEMENTING MAJOR LEAGUE BASEBALL'S NEW
\end{tabular} HEALTH AND INJURY TRACKING SYSTEM (HITS)}

${ }^{1}$ Keshia M Pollack, ${ }^{2}$ John D'Angelo, ${ }^{3}$ Gary Green, ${ }^{4}$ Stan Conte, ${ }^{5}$ Stephen Fealy, ${ }^{2}$ Chris Marinak, ${ }^{6}$ Edward McFarland, ${ }^{7}$ Frank Curriero. ${ }^{1}$ Johns Hopkins Centre for Injury Research and Policy, Baltimore, MD, USA; ${ }^{2}$ Major League Baseball; ${ }^{3}$ Pacific Palisades Medical Group; ${ }^{4}$ Conte Injury Analytics; ${ }^{5}$ Hospital for Special Surgery; ${ }^{6}$ Johns Hopkins School of Medicine; ${ }^{7}$ Johns Hopkins Bloomberg School of Public Health

\subsection{6/injuryprev-2016-042156.748}

Background In 2010, Major League Baseball (MLB) and the Major League Baseball Players Association reached an agreement on an electronic medical records and injury tracking system. One goal of the system was to identify and monitor injury trends to better optimise player health and safety.

Methods Development of the surveillance system, HITS (Health and Injury Tracking System) database involved reviewing existing sports injury surveillance systems, consulting with the certified athletic trainers who record player injuries and illnesses, as well as experts who had experience with existing sports databases, and team physicians.

Results HITS includes all players from the Major and Minor Leagues rather than a sample of players from certain teams. The HITS system has a unique identifying number for each player; thus, data can be deterministically linked across various databases. Linking the data allows for investigation of injuries with regard to other key measures of exposure such as pitch count and number of hits, surgical outcomes data, personnel records to calculate cost data, and demographic data. Events included in HITS are any injury or physical complaint sustained by a player that affects or limits participation in any aspect of baseball-related activity, such as a game, practice, or warm up. Analysis of the HITS data has explored leading body parts injured during play including the hamstring, knee, shoulder, and head; select positions such as catchers; and key activities such as sliding.

Conclusions The implementation of HITS has advanced sports injury research overall and professional baseball research in particular. The richness of HITS is unprecedented and creates an opportunity to identify and monitor injury trends in baseball, and conduct epidemiologic research to better understand player risk, and optimise player health and safety through possible rules changes, equipment modifications, or medical education.

\section{INJURY INCIDENCE AND LOCATION OF ACUTE INJURIES AT A NATIONAL LEVEL IN SEVEN POPULAR SPORT}

${ }^{1}$ Malin Åman, ${ }^{2}$ Magnus Forssblad, ${ }^{1}$ Karin Henriksson-Larsén. ${ }^{1}$ GIH, the Swedish School of Sport and Health Sciences, Sweden; ${ }^{2}$ Stockholm Sport Trauma Research Centre, Karolinska Institute, Sweden

\subsection{6/injuryprev-2016-042156.749}

Background Acute injuries in sports are still a problem and the aetiology of injuries in different sports, at a national level, are limited. The aim of the study was to describe the body location of acute injuries in seven sports, which has previously been identified as high-risk sports with respect to incidence of acute injury and severity of injuries, at a national level.

Methods An epidemiological study was performed using insurance data between years 2006-2013. Sports was motorcycle, 